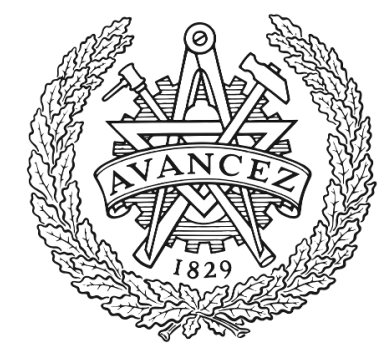

CHALMERS

UNIVERSITY OF TECHNOLOGY

\title{
Modulation and detection for multicore superchannels with correlated phase noise [Invited]
}

Downloaded from: https://research.chalmers.se, 2023-04-26 14:12 UTC

Citation for the original published paper (version of record):

Agrell, E., Alfredsson, A., Puttnam, B. et al (2018). Modulation and detection for multicore superchannels with correlated phase noise [Invited]. Proc. Conference on Lasers and Electro-Optics (CLEO), Part F94-CLEO_SI 2018. http://dx.doi.org/10.1364/CLEO_SI.2018.SM4C.3

N.B. When citing this work, cite the original published paper. 


\title{
Modulation and Detection for Multicore Superchannels with Correlated Phase Noise
}

\author{
Erik Agrell ${ }^{1}$, Arni Alfredsson ${ }^{1}$, Benjamin J. Puttnam ${ }^{2}$, \\ Ruben S. Luís ${ }^{2}$, Georg Rademacher ${ }^{2}$, and Magnus Karlsson ${ }^{3}$ \\ ${ }^{1}$ Dept. of Electrical Engineering, Chalmers University of Technology, SE-41296 Gothenburg, Sweden \\ ${ }^{2}$ Photonic Network System Laboratory, NICT, 4-2-1 Nukui-Kitamachi, Koganei, Tokyo 184-8759, Japan \\ ${ }^{3}$ Dept. of Microtechnology and Nanoscience, Chalmers University of Technology, SE-41296 Gothenburg, Sweden \\ agrell@chalmers.se
}

\begin{abstract}
SDM fibers offer new opportunities and challenges for joint transmitter and receiver processing. We review multidimensional modulation and detection theory, describe algorithms for phase tracking and detection over spatial superchannels, and present some experimental results.
\end{abstract}

OCIS codes: $\quad 060.1660$ Coherent communications, 060.2330 Fiber optics communications, 060.4080 Modulation.

\section{Introduction}

In the continuous struggle to avoid, or at least defer, the looming "capacity crunch," the spotlight is now on spacedivision multiplexing (SDM) via multicore or multimode optical fibers [1]. This technology opens up the opportunity to form spatial superchannels, over which data is encoded and decoded jointly. The corresponding signal-space description moves from the traditional four dimensions (two polarizations with two quadratures each) [2] to tens or hundreds of dimensions. To take full advantage of this multidimensional geometry, novel transmitter and receiver techniques are required. In this presentation, we will exemplify such techniques for the purpose of modulation and phase noise mitigation.

\section{Multidimensional modulation}

Modulation format design is, in geometric terms, the science of placing $M$ constellation points in $N$ dimensions. In a small number of dimensions, the individual point locations have been optimized for different metrics, including minimum distance [2,3], achievable information rate [4], and nonlinearity tolerance [5]. With more dimensions, however, the constellations need to have a regular structure to simplify implementation. Since at any fixed spectral efficiency, the number of points grows exponentially with the dimension, the storage and decoding complexity quickly get out of hand if each point is to be processed individually. Instead of look-up tables, high-dimensional constellations need to be generated and processed using algebraic rules. Structured constellations that have been studied in optical communications include product constellations, generalized pulse-position modulation, lattices (of which constellations formed by set partitioning is a popular special case), and binary block codes $[6,7]$.

In this presentation, we apply binary block codes for the design of constellations across multiple spatial dimensions. Thus, instead of applying coding across consecutive time slots as in standard applications of forward-error-correction (FEC) coding, the whole codeword is transmitted instantaneously, in the same time slot. This has obvious benefits in low-latency applications such as remote sensing and control, autonomous traffic systems, and tactile Internet. A low pre-FEC bit error rate (BER) also facilitates receiver processing using decision feedback.
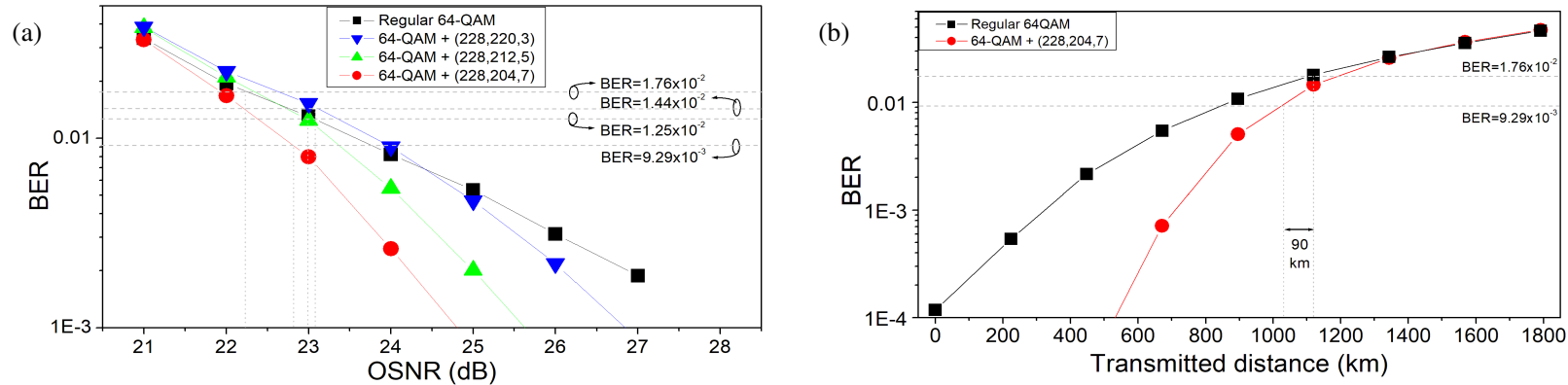

Fig. 1: Multidimensional modulation over (a) a single span of $30 \mathrm{~km}$ and (b) a recirculating loop. 
(a)

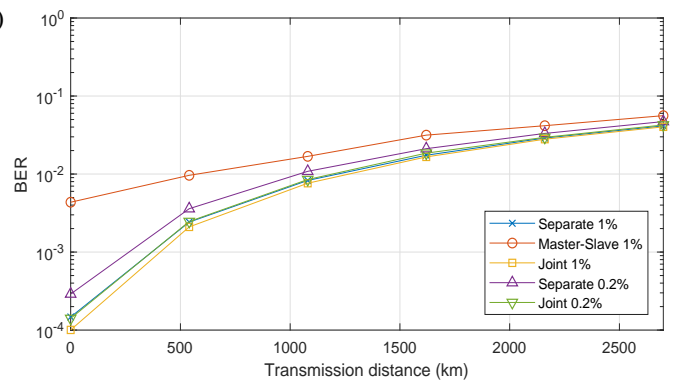

(b)

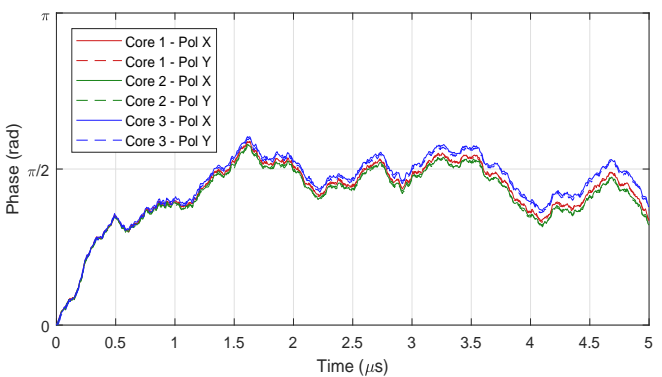

Fig. 2: (a) The BER with various phase-noise mitigation strategies. (b) The phase noise of two polarizations in three cores.

We performed transmission experiments over 19-core fiber at 25 Gbaud. The considered formats transmit 12, 11.58, 11.16, and 10.74 bits per core, where the largest constellation is independent dual-polarization 64-ary quadrature amplitude modulation (DP-64QAM) in each core and the other three are 76-dimensional modulation formats (four dimensions per core) designed using binary linear block codes. The details of the experimental setup and the format constructions are reported in [8]. In Fig. 1(a), it is shown that the format with 10.74 bits per core gains about 2.7 $\mathrm{dB}$ over DP-64QAM at a target bit error rate (BER) of $2 \cdot 10^{-3}$. This gain comes as the expense of $10.5 \%$ smaller throughput. On the other hand, if an outer FEC code is applied to bring the overall BER down to $10^{-15}$, then it is more beneficial (by 0.6-0.8 dB in required OSNR) to combine a large constellation (DP-64QAM) with a low-rate outer code than vice versa. This is shown in Fig. 1 by applying different pre-FEC BER thresholds to each constellation, chosen from [9, Tab. I] in such a way that the overall information rate is approximately 60 Gbps (9.6 bits per core) in all four cases. The long-haul transmission experiment in Fig. 1(b) confirms this trend, giving $90 \mathrm{~km}(9 \%)$ longer reach for 64-QAM than the format with 10.74 bits per core at the same information bit rate.

\section{Multidimensional phase noise mitigation}

To process spatial channels jointly is beneficial on the receiver side too. Specifically, if a common local oscillator is used for all channels, which is usually the case, the phase noise of different channels will be strongly correlated [10]. This correlation can be utilized to either reduce complexity or improve performance compared with tracking the phase separately on each channel. In this work, all strategies are implemented using a pilot-aided algorithm that we developed. It performs phase-noise-aware detection, and the phase-noise statistics can be estimated using observations from an arbitrary number of channels. Thus, in case of separate recovery, the algorithm is applied independently on each channel, whereas for joint recovery, it uses observations from all available channels. To implement a master-slave scheme, estimates are obtained from phase-noise statistics that are gathered from observations on a single channel.

In Fig. 2(a), results for the different phase-recovery strategies and pilot rates of $1 \%$ and $0.2 \%$ are shown in terms of BER as a function of distance for a DP-16QAM 3-core transmission experiment that we performed. In the case of $1 \%$ pilot rate, a modest improvement of about $50 \mathrm{~km}$ increase in transmission reach is observed by performing joint compared to separate recovery. For $0.2 \%$ pilot rate, a larger gain of about $150 \mathrm{~km}$ increase in reach is seen. Moreover, joint recovery with $0.2 \%$ pilot rate has comparable performance to separate recovery with $1 \%$ pilot rate, indicating that the pilot rate can be significantly reduced through joint processing. Finally, the master-slave scheme sees a severe penalty from the lack of perfect phase-noise correlation across the channels. This is mainly due to the presence of residual frequency offsets between the cores, as illustrated in Fig. 2(b).

\section{References}

1. E. Agrell et al., "Roadmap of optical communications," Journal of Optics 18, 063,002 (2016).

2. E. Agrell and M. Karlsson, "Power-efficient modulation formats in coherent transmission systems," J. Lightw. Technol. 27, 5115-5126 (2009).

3. E. Agrell, "Database of sphere packings," Online: http://codes.se/packings.

4. I. B. Djordjevic, "Spatial-domain-based hybrid multidimensional coded-modulation schemes enabling multi-Tb/s optical transport," J. Lightw. Technol. 30, 3888-3901 (2012).

5. C. Häger, A. Graell i Amat, A. Alvarado, and E. Agrell, "Design of APSK constellations for coherent optical channels with nonlinear phase noise," IEEE Trans. Commun. 61, 3362-3373 (2013).
6. D. S. Millar et al., "High-dimensional modulation for coherent optical communications systems," Opt. Exp. 22, 8798-8812 (2014).

7. M. Karlsson and E. Agrell, "Multidimensional optimized optical modulation formats," in "Enabling Technologies for High Spectral-Efficiency Coherent Optical Communication Networks," X. Zhou and C. Xie, eds. (Wiley, 2016), ch. 2.

8. B. J. Puttnam et al., "High capacity transmission systems using homogeneous multi-core fibers," J. Lightw. Technol. 35, 1157-1167 (2017).

9. L. M. Zhang and F. R. Kschischang, "Staircase codes with $6 \%$ to 33\% overhead," J. Lightw. Technol. 32, 1999-2002 (2014).

10. M. D. Feuer et al., "Joint digital signal processing receivers for spatial superchannels," IEEE Photon. Technol. Lett. 24, 1957-1960 (2012). 